\title{
Modernes Prozessleitsystem lässt Wasser in Oberösterreich verlässlich fließen
}

Dass in unserem Lebensumfeld Wasser in jeder Menge und zu jeder Zeit aus der Leitung kommt, scheint selbstverständlich. Damit dieses Naturelement stets verfügbar ist, bedarf es am anderen Ende der Wasserleitung eines enormen Aufwandes - besonders auf der technischen Seite. Durch den Einsatz moderner Prozessleitsystemekann der Wasserversorger die Verfügbarkeit und Qualität unseres Trinkwassers immer auf dem gleich hohen Niveau halten, ohne dabei die Wirtschaftlichkeit aus den Augen zu verlieren. Die beiden Unternehmen ETM professional control $\mathrm{GmbH}$ und deren OEM-Partner Rittmeyer AG bündeln ihr langjähriges Know-how im Prozessleitsystem RITOP ${ }^{\circledR}$. Erfolgreich eingesetzt wurde RITOP $^{\circledR}$ zuletzt vom oberösterreichischen Wasserversorger WDL.

\section{Erfolgreiche Partnerschaft seit 1.000 Lizenzen}

Auf der einen Seite steht die Schweizer Rittmeyer AG als führender Anbieter für Lösungen in der Versorgung mit Wasser, Gas und Elektrizität. Auf der anderen Seite steht mit ETM professional Control $\mathrm{GmbH}$ ein führender Spezialist aus Österreich für modernste Prozessleittechnik. Vor nunmehr 1.000 Lizenzen hatten sich die Schweizer entschlossen, als Grundlage für das Prozessleitsystem RITOP $^{\circledR}$ das Scada-System PVSS von ETM einzusetzen. Mit dieser Entscheidung manifestierte sich RITOP $^{\circledR}$ als durchgängiges, objektorientiertes System mit Langzeitarchivierung sowie Verdichtungs- und Bilanzierungsfunktionen als das führende Leitsystem im Versorgungsbereich. RITOP $^{\circledR}$ punktet vor allem durch seine umfangreichen Protokolliermöglichkeiten, seine effiziente Online- bzw. Offline-Konfiguration und seine Systemoffenheit. Mit optimierten Branchenpaketen für Wasser- und Energieversorgung, Kraftwerke und Hydrografie sowie Verfahrensund Umwelttechnik ist RITOP ${ }^{\circledR}$ optimal auf die Bedürfnisse der Kunden abgestimmt.

\section{Führend in Österreich}

Auch in Österreich wurden von der hiesigen Niederlassung in Wien schon zahlrei- che Projekte erfolgreich realisiert. Geschäftsführer Friedrich Lehr von der Rittmeyer GmbH: ,Wir haben in den letzten Jahren in Österreich die wichtigsten Wasserversorgungsanlagen mit unserem Leitsystem RITOP ${ }^{\circledR}$ ausgestattet. Bei unseren Kunden punkten wir vor allem durch die Flexibilität und Offenheit des Systems. So können wir sowohl Neuanlagen als auch Modernisierungsprojekte auf den letzten Stand der Technik bringen. Das bringt uns vor allem dann einen großen Nutzen, wenn historisch gewachsene Peripherien eingebunden werden müssen.

Ein weiterer großer Vorteil von RITOP ${ }^{\circledR}$ auf Basis von PVSS ist das Large-ScaleKonzept. In allen permanent oder temporär verbunden Systemen ist der transparente Zugriff auf sämtliche Daten (Historie, Online-Daten, Alarme, Parameter etc.) möglich. Das beinhaltet Bedienung, Anlagenbilder, Alarmquittierung und Engineering. Damit sind wir in der Lage, sowohl weit entfernte als auch schwer erreichbare Außenanlagen sicher und wirtschaftlich einzubinden. Mit ein Grund, dass einer der größten Wasserversorger Oberösterreichs auf RITOP $^{\circledR}$ von Rittmeyer vertraut."

\section{Tafelwasser für Oberösterreich}

Die oberösterreichische WDL - Wasserdienstleistungs $\mathrm{GmbH}$ ist für die Wasserversorgung von über 125.000 Einwohnern Oberösterreichs und der angrenzenden bayerischen Stadt Burghausen verantwortlich. Rund um die Uhr werden die 36 angeschlossenen Gemeinden und Städte je nach Wunsch mit frischem Wasser volloder teilversorgt. Das Wasser kommt ausschließlich aus WDL-eigenen Brunnen, Hochbehältern und Leitungen und wird an Übergabestellen an die Kommunen übergeben. Das schadstofffrei gewonnene Wasser muss weder aufbereitet noch bestrahlt oder gechlort werden. Dafür sorgen ideale geologische Verhältnisse und von menschlichen Einflüssen weitgehend geschützte Gebiete.

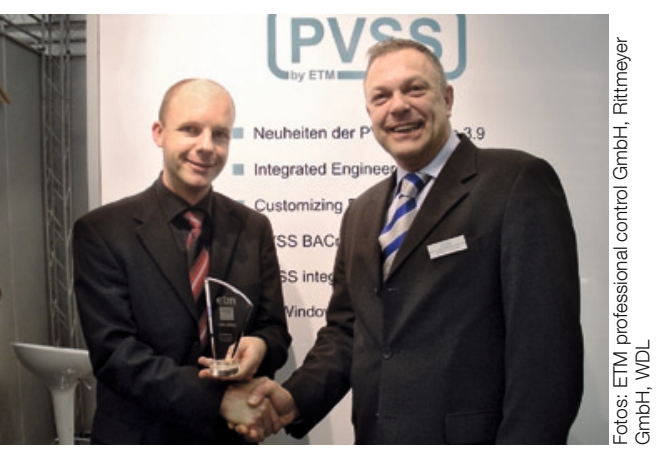

Abb. 1: Übergabe der Auszeichnung 1000.PVSS Lizenz: Gerhard Nigg, Leiter Entwicklung bei Rittmeyer AG (I.) und Bernhard Staufer, Director Sales \& Marketing bei M professional control GmbH

\section{Versorgungsnetz effizient} überwachen und steuern

Das weit verteilte Versorgungsgebiet basiert auf einem dezentralen Versorgungskonzept. Dem entsprechend ist auch die Organisation mit je einem Bereichsleiter und einem Betriebsleiter pro Versorgungsregion ausgelegt. Da Versorgungssicherheit bei WDL oberste Priorität hat, werden alle Bereichs- und Betriebsleiter über das Leitsystem von den anderen Versorgungsbereichen informiert. Das hat den Vorteil, dass im Störungsfall der Bereitschaftsdienst mit ausreichend Daten versorgt wird, diese aber trotzdem sehr differenziert aufbereitet sind. So kann der Bereitschaftsdiensthabende sofort feststellen, ob es sich um einen niedrigen Wasserstand handelt, dessen Ursache erst am nächsten Tag behoben werden muss, oder ob eine ganze Mannschaft vor Ort geschickt werden muss, um einen sofortigen Wasserengpass wegen eines technischen Defekts zu beheben. Selbstverständlich kann jeder diensthabende Bereichsleiter auf jedes angeschlossene System zugreifen.

\section{Versorgungssicherheit durch flexibles Leitsystem}

Vor drei Jahren entschloss sich WDL, ihre Leitsystemkonfiguration $\mathrm{zu}$ vereinheitlichen. Bis dahin waren nahezu alle Bereiche mit unterschiedlichen Leitsystemen ausgestattet. In den vorangegangenen Jahren konnte man Erfahrungen mit jedem einzel- 


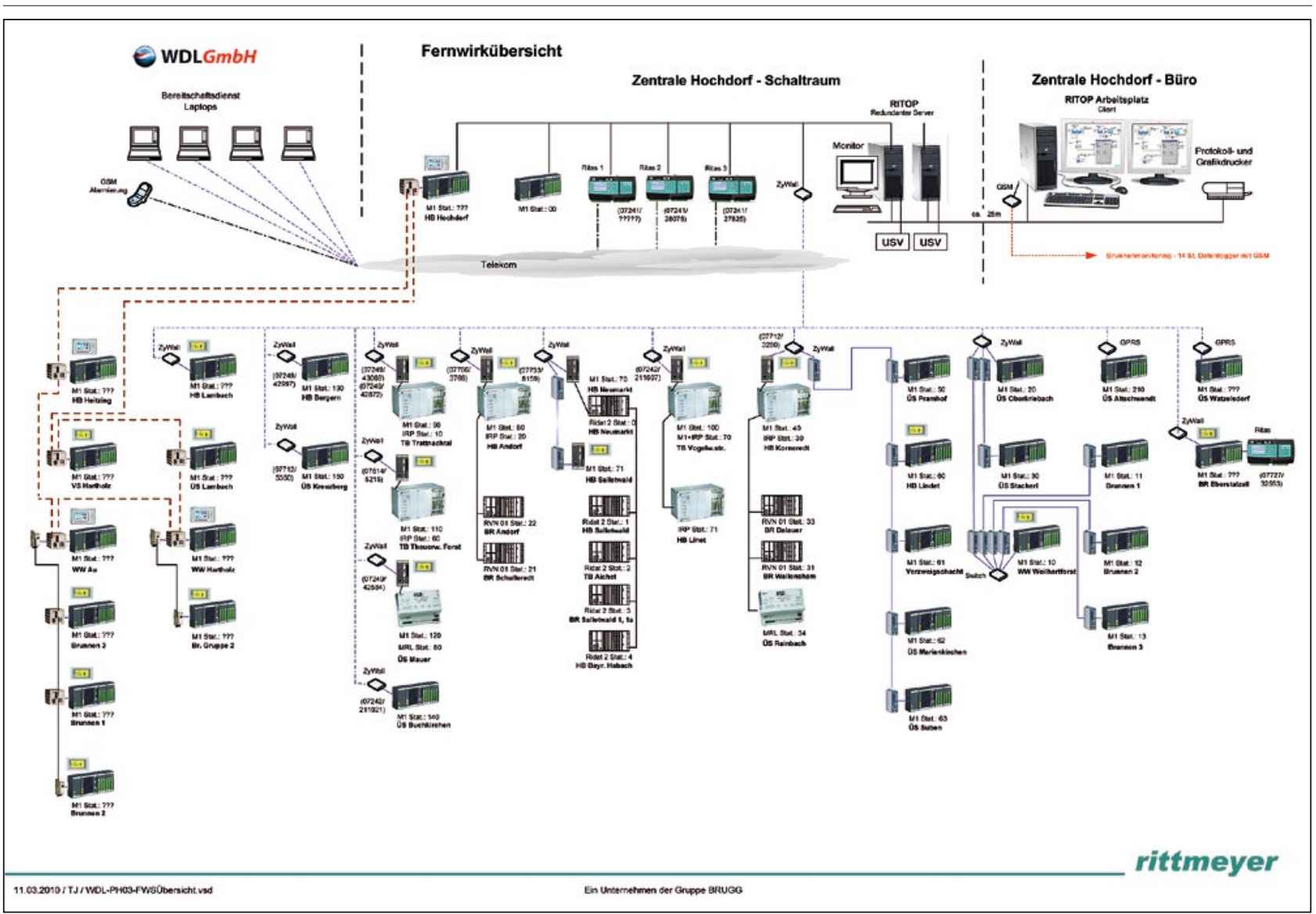

Abb. 2: WDL - Fernwirkübersicht.

nen System sammeln, stellte Stärken und Schwächen gegenüber und entschloss sich letztendlich für das weltweit erprobte RITOP ${ }^{\circledR}$ von Rittmeyer. „Ein wesentlicher Entscheidungsfaktor war die laufende Weiterentwicklung des Leitsystems, die gerade bei den individuellen Anforderungen unserer dezentralen Anlagen zum Tragen kommt", begründet WDL-Geschäftsführer DI Andreas Pfusterer die Systementscheidung. „Wir müssen in allen Anlagen unterschiedliche Maßnahmen setzen. Da ist es besonders wichtig, dass die hereinkommenden Informationen so aufbereitet sind, dass in der Bereitschaft, die in der Regel aus einer Person besteht, sofort die nötigen Entscheidungen stressfrei getroffen werden können. Hier punktet RITOP ${ }^{\circledR}$ durch seine Flexibilität und seiner Anpassungsfähigkeit auf unsere Anforderungen."

Nach der Entscheidung für Rittmeyer wurde vom führenden Anbieter für Lösungen in der Wasser- und Energieversorgung auf Basis des Angebotes ein LeittechnikKonzept erstellt, das in enger Zusammenarbeit mit WDL Step-by-Step in einem Jahr umgesetzt wurde. Die Implementierung von RITOP ${ }^{\circledR}$ erwies sich als besonders einfach. Nach einem kurzen Parallelbetrieb,

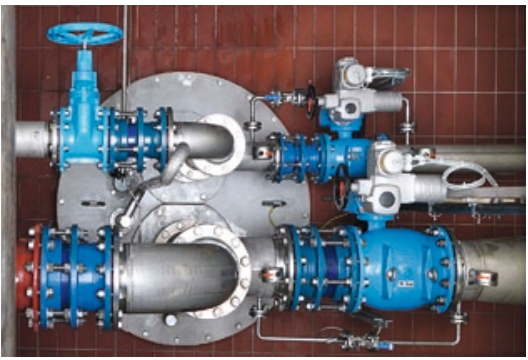

Abb. 3: Brunnenkopf.

in dem die Peripherie an das neue System angepasst wurde, konnte problemlos umgeschaltet werden.

\subsection{PVSS-Fact Objektorientierung}

Dank dem durchgängigen objektorientierten Ansatz von PVSS wird ein hoher Grad an Standardisierung erzielt. Angefangen bei der Planung bis zur Inbetriebnahme bis hin zum Betrieb. Darüber sind auch die Qualifikationen des Betriebspersonals gezielter einsetzbar und bieten mehr Komfort für Anwender und Projektanten. Dies wirkt sich besonders bei den Engineeringkosten aus: Einsparungen bis zu $70 \%$ sind möglich.

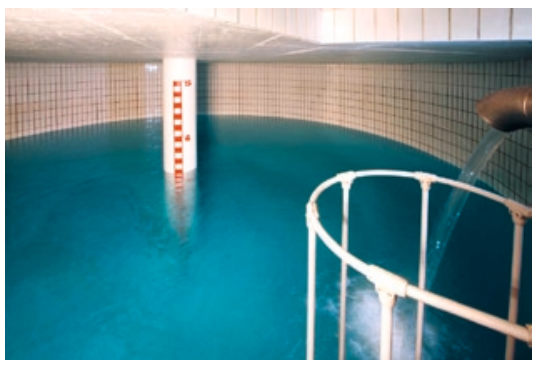

Abb. 4: Hochbehälter Arndorf.

\section{Kommunikation „State-of-the-Art"}

In einem weit verteilten System ist die Kommunikation zwischen den einzelnen Anlagenteilen und dem Leitsystem immer wieder eine Herausforderung. „Die Fernwirkstruktur bei WDL entspricht dem neuesten Stand der Technik und beinhaltet die modernsten Komponenten, die in der Telekommunikation derzeit am Markt verfügbar sind“ erläutert Alois Doninger, Rittmeyer-Vertriebsleiter für Oberösterreich und Salzburg. „Entsprechend der Sicherheitsanforderungen von WDL basiert das Konzept auf kabelgebundenem Internet (Ethernet TCP/IP) mit VPN-Tunnels, red- 


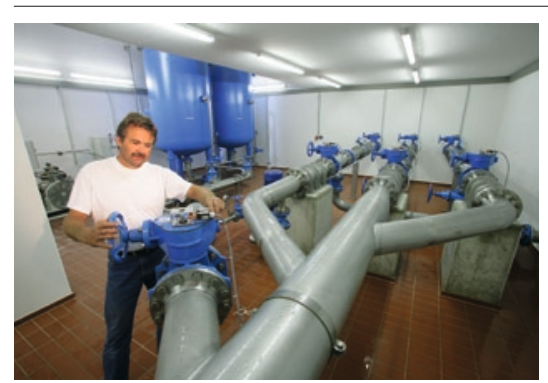

Abb. 5: Wasserversorgung für die bayerische Stadt Burghausen.

undanten Servern in der Zentrale sowie Notbetriebsebenen und Notsteuerungen in der Peripherie."

\subsection{PVSS-Fact Fernwirkfähigkeit}

Das auf Fernwirkfähigkeit ausgerichtete System entspricht nicht nur genau den Kommunikationsvorgaben von WDL sondern bietet darüber hinaus noch systemintern ein verlässliches Datensicherheitskonzept. Um bei einem Leitungsausfall einem Datenverlust weitgehend vorzubeugen, werden die Prozessvariablen mit Zeitstempel, Qualitätsinformationen, Default-Werten, Quelle etc. versehen. Weiters erfolgen Datenbank-Synchronisation und -Abgleich automatisch. Die Übertragungssicherheit wird durch Verschlüsselung garantiert und die Funktion „Message-Compression" reduziert den Bandbreitenbedarf - d.h., es kommt zu keinem Stau auf der Datenautobahn. Ne- ben der Anbindung an die Steuerungsebene stellt RITOP ${ }^{\circledR}$ auch Daten für WDLinterne Systeme zur Verfügung. So werden Auflagen des Landes Oberösterreich wie auch eigene, höhere Auflagen direkt im System verwaltet und über Standardschnittstellen mit dem Umweltnetzwerk des Landes bzw. mit dem Betriebsführungssystem der WDL verknüpft. Gerade bei Regressforderungen ist die lückenlose Betriebsdokumentation von Wassergüte und Umwelteinflüssen ein unabdingbarer Beweisfaktor.

\section{Anlageneffizienz und Verfügbarkeit gesteigert}

„Mit dem Einsatz von RITOP ${ }^{\circledR}$ konnten wir den internen Prozessablauf absolut effizient gestalten. Nun kommen die Informationen aus einem wesentlich tieferen Teil der Anlagen. Das verkürzt die Fehlersuche, grenzt die Ursachen schon frühzeitig ein, ermöglicht einen koordinierten Personaleinsatz und reduziert den materiellen Aufwand im Service- und Wartungsmanagement" zieht Andreas Pfusterer positiv Bilanz. Und die kann sich sehen lassen: mit insgesamt 7 Personen werden alle Anlagen betreut. Und dazu gehören die Stadt Wels samt Umgebung, das Gebiet Schärding bis Neumarkt, die Regionen Kremstal, Vorchdorf, Sattledt und das eingangs erwähnte Burghausen am bayerischen Innufer sowie zahlreiche weitere Gemeinden.

\section{WDL in fact (2007/2008)}

Fakt. Trinkwasser

Versorgte Einwohner Trinkwasser

Versorgte Einwohner Abwasser

Abwasseranlagen

Mitarbeiter (in FTE)

Verträge mit Kommunen

Wasserleitungsnetz (exkl. Hausanschlüsse)

Kanalnetz (exkl. Hausanschlüsse)

Kanalservice
Wasserversorgungsanlagen
8,2 Mio. $\mathrm{m}^{3}$

30.000

12

4

43

45

$250 \mathrm{~km}$

$100 \mathrm{~km}$

$1.000 \mathrm{~km} / \mathrm{a}$
178.000

\section{RITOP ${ }^{\circledR}$ in fact}

- Redundantes Prozessleitsystem mit je 2 USV-gestützten Servern

- 1 zentraler Arbeitsplatz mit Monitoren, Protokoll- und Grafikdruckern, Modemanbindung (GSM) an 14 Datenlogger

- 4 dezentrale Arbeitsplätze via Ethernet TCP/IP (Bereitschaftsdienst-Laptops)

- Alarmausgabe mittels 4 Alarmservern (RITAS)

- Anbindung über GSM an ein Alarmierungs-Mobiltelefon.

- 27 Fernwirk- und Automatisierungsstationen RIFLEX M1 in den Außenobjekten, Anbindung via Ethernet TCP/IP
Im Gegensatz zu anderen Wasserversorgern ist die Organisationsstruktur dank der dezentralen Hierarchie von WDL besonders schlank. Da konnte es passieren, dass die Zentrale von einem Tag auf den anderen örtlich übersiedeln musste. Hier zeigte sich die Flexibilität von RITOP $^{\circledR}$ in besonderer Weise. Unmittelbar nach dem kurzen Wiederaufbau war das System betriebsbereit. Ebenso kein Thema ist die Erweiterung von Anlagenteilen. WDL übernimmt immer öfter die Wasserverteilung innerhalb von Gemeinden. Damit fällt auch die dortige Sicherstellung des Lebensmittels Nummer 1 in den Verantwortungsbereich der Energie-AG-Tochter.

\subsection{PVSS-Fact Client-Server Architektur}

Dank dieser System Architektur ist eine nahezu uneingeschränkte Skalierung gegeben: vom Einzelplatz- bis zum verteilten redundanten System in unterschiedlichsten Varianten. So können unterschiedliche Betriebssysteme (Windows, Linux, Solaris) vermischt und damit auch unabhängig erweitert werden. PVSS ist ein Multiuser- und Multitaskingsystem, dessen Aufbau aus funktionsbezogenen Managern besteht. Die interne Kommunikation erfolgt auf TCP/IP und ist vollkommen ereignisorientiert sowie telegrammbasiert. Abgesetzte Client-Arbeitsplätze können via Modem angekoppelt werden. Damit sind mit PVSS ausgerüstete Anlagen auch zukünftig jederzeit erweiterbar.

WDL stehen mit RITOP $^{\circledR}$ folgende Funktionen zur Verfügung:

- vollgrafische Visualisierung,

- objektorientierte Prozessführung,

- umfangreiche Protokolliermöglichkeiten (Meldepanel, Betriebsstundenprotokoll, Login-/Logoutprotokoll),

- umfangreiche Alarmverarbeitung

- umfassendes Prozessdatenarchiv,

- vielfältige Tabellen- und Grafikausgabe (Trendingeinblendungen in Prozessbildern, Tagesprotokolle, Monatsprotokolle, Jahresprotokolle),

- flexible Registrierfunktion,

- Sollwertkurven/Funktionskurven. 